Théologiques

Théologiques

\title{
Ritualités dans la (dis-)continuité
}

Agencements et petits arrangements de femmes françaises converties à l'islam

\section{Amélie Puzenat}

Volume 21, numéro 2, 2013

La conversion

URI : https://id.erudit.org/iderudit/1028467ar

DOI : https://doi.org/10.7202/1028467ar

Aller au sommaire du numéro

\section{Éditeur(s)}

Faculté de théologie et de sciences des religions, Université de Montréal

ISSN

1188-7109 (imprimé)

1492-1413 (numérique)

Découvrir la revue

\section{Citer cet article}

Puzenat, A. (2013). Ritualités dans la (dis-)continuité : agencements et petits arrangements de femmes françaises converties à l'islam. Théologiques, 21(2), 179-199. https://doi.org/10.7202/1028467ar

\section{Résumé de l'article}

La réappropriation de rites de passage islamiques que sont le mariage, l'aqîqa et la circoncision, par des femmes converties à l'islam, marque leur inscription dans un nouveau groupe d'appartenance. La mise en conformité de rituels traditionnels avec les exigences du renouveau islamique contemporain, tel qu'il prend forme en France, en sus de la négociation avec une culture héritée non islamique qu'elles mettent en oeuvre, s'apparente à un agencement de l'entre-deux qui participe, d'une part, à leur inclusion sociale au sein de la communauté de croyants et, d'autre part, à produire une nouvelle éthique religieuse. Toutefois, à la suite d'une volonté marquée de dé-culturer le religieux, de se distancier de rites perçus comme coutumiers et non valides sur le plan religieux, la fonction de rassemblement des ritualités se limite à un entre-soi religieux, car une mise à distance, voire une mise à l'écart des familles s'opère. 


\title{
Ritualités dans la (dis-)continuité
}

\section{Agencements et petits arrangements de femmes françaises converties à l'islam}

\author{
Amélie Puzenat" \\ Sociologie \\ Université catholique de l'Ouest (Angers, France)
}

Nous assistons aujourd'hui au renouvellement des études socioanthropologiques sur les phénomènes de conversions religieuses. Que celles-ci envisagent la conversion religieuse comme marqueur de l'individualisation religieuse contemporaine, où l'individu exprime des agentivités spécifiques en la matière (Jensen 2006), ou bien qu'elles approchent ce phénomène comme marqueur de la globalisation de certains mouvements religieux (Gooren 2007), peu s'intéressent aux rites de passage entretenus par les convertis, hormis au rituel spécifique de conversion. Pourtant, les religions ne peuvent se passer des rites formalisés qui leur donnent corps, perpétuent le groupe et sa mémoire dans le temps et rappellent la présence de l'au-delà dans l'ici-bas (Piette 2002).

À travers l'étude de rites célébrant la fécondité et la naissance, nous décrirons ici la production de formes religieuses propres aux femmes converties à l'islam, celle-ci étant révélatrice aussi bien de leur agentivité que de leur mise en conformité avec une idéologie religieuse globalisée et adaptée localement, celle du renouveau islamique. Les mises en scène rituelles que ces femmes développent définissent une identité individuelle et collective entre tradition et modernité, dans un contexte français en partie

Amélie Puzenat est Maître de Conférences en sociologie à l'Université catholique de l'Ouest, elle est également membre du laboratoire de recherche Espaces et Sociétés de l'Université d'Angers. Ses recherches portent sur les couples "mixtes » francomaghrébins et les conversions à l'islam, en France. Elle a notamment publié (2013) "Déculturaliser l'interculturalité: des couples franco-maghrébins à l'épreuve du renouveau islamique» dans F. Dervin, dir., Relations intimes interculturelles, Paris, Editions des archives contemporaines.

C) Revue Théologiques 2013. Tout droit réservé. 
sécularisé où l'islam est socialement religion de "minorités " (Simon et Tiberj 2013). Nous avons fait le choix de nous arrêter sur l'étude de trois rites de passage: le mariage, la circoncision du jeune garçon et l'aqîqa, cérémonie organisée à la naissance de l'enfant, lors de laquelle est également parfois pratiquée la circoncision. Tous participent d'un changement de statut social de l'individu, en fonction de son sexe, dès sa venue au monde jusqu'à l'âge adulte. Hormis le rite du mariage, qui peut avoir lieu plusieurs fois au cours de la vie, les rites de passage que représentent l'aqîqa et la circoncision ne se produisent, en principe, qu'une seule fois dans la vie de l'individu.

Détailler ces formes rituelles révèle non seulement les modalités de construction sociale du genre à l'œuvre, mais également la négociation qui s'opère entre un islam de tradition, celui souvent pratiqué par les beauxparents musulmans des femmes converties, et un islam d'élection, celui du renouveau islamique dont elles ont fait le choix et où le travail d'épuration du rite est visible (Roy 2008). Les agencements rituels, bien que travaillés par les trajectoires personnelles et familiales, rendent compte de nouvelles modalités spécifiques au renouveau islamique dont nous donnerons une tentative de cadrage dans une première partie. Ils s'inscrivent dans un islam pratiqué par la jeune génération de musulmans en France, ceux que Arslan (2010) nomme "les born again Muslim».

De surcroît, ces rites de passage, que les femmes converties à l'islam revisitent, travaillent à la reproduction sociale du groupe d'appartenance. Leur étude dévoile ainsi les enjeux autour du legs identitaire à la descendance. Ce qui nous intéresse, dans le cadre de cet article, est donc, non seulement de décrire le type d'islam mi-perpétué, mi-réinventé, à travers les formes rituelles que ces femmes développent, mais également de restituer les différentes négociations qui s'opèrent autour de l'héritage familial. La particularité du contexte post-migratoire dans lequel elles s'inscrivent, même si elles ne sont qu'indirectement concernées, fait du religieux un moyen de préserver un héritage, une mémoire collective et familiale, et de reconnaître les origines extra-nationales des enfants.

\section{Présentation des femmes converties à l'islam et de l'islam des femmes converties}

Les données présentées ici ont été recueillies dans le cadre d'un doctorat de sociologie portant sur les redéfinitions identitaires liées aux conversions à 
l'islam en France, notamment en région parisienne. Dans le cadre de cette présentation, nous nous concentrerons sur l'étude de quarante-sept entretiens menés auprès de femmes de nationalité française converties à l'islam, de parents français non immigrés. Avant de se convertir à l'islam, ces femmes étaient soit de tradition chrétienne, généralement peu ferventes, soit athées. Lorsque nous les avons interviewées, la plupart vivaient en couple avec leurs enfants, quelques-unes vivaient en couple sans enfant ou étaient divorcées (avec ou sans enfant), et quatre vivaient seules sans enfants. Les conjoints «musulmans» des femmes mariées sont soit issus de l'immigration maghrébine (17), soit immigrés maghrébins (14). L'enquête compte également cinq conjoints provenant d'Afrique subsaharienne.

La recherche s'est déroulée de 2006 à 2009, au sein de réseaux de femmes converties liées virtuellement ou implantées spatialement en banlieue parisienne, notamment en Seine-Saint-Denis. Ainsi, la plupart de ces femmes qui ne sont pas originaires de la banlieue parisienne, mais proviennent de milieux urbains de province et quelques fois de milieux ruraux, résident au sein de vastes zones d'habitats sociaux. Globalement, nous avons affaire à une majorité de couples ou de femmes vivant en banlieue qui relèvent de la catégorie «classe moyenne» et, dans une moindre mesure, des «classes populaires».

Un autre élément important à prendre en compte, pour comprendre les ritualités développées par ces femmes converties, est leur inscription dans un type d'islam contemporain spécifique. Le renouveau islamique n'est pas nécessairement l'apanage des femmes converties à l'islam, mais il reflète majoritairement celui que prônent les femmes rencontrées lors de notre étude. Sans chiffres ni statistiques nationales précises, nous ne pouvons donc dire en quelle mesure il est représentatif du «croire» islamique des femmes converties. Il s'agit simplement d'une tendance que nous avons dégagée, minoritaire peut-être. Toutefois, dans notre étude, nous traitons d'un islam marqué du sceau du renouveau islamique ou de ce que les chercheurs qualifient également de processus de réislamisation ${ }^{1}$.

1. Nous faisons, dans ce texte, le choix de parler de réislamisation et de renouveau islamique, mais non d'islamisme ou de réformisme islamique, le premier terme étant connoté politiquement et péjorativement (Seniguer 2012) et le second prêtant, selon nous, à confusion. Le réformisme islamique contemporain peut, en effet, aussi bien renvoyer à un mouvement réformiste conservateur qu'à un mouvement réformiste libéral, du point de vue de l'interprétation doctrinale (Zhegal 2010). Et, bien que le terme de réislamisation soit aujourd'hui remis en cause, car supposant, en amont, un processus de "désislamisation " (Saint-Lary et Samson 2011), nous faisons le choix de conserver ce terme plutôt que de parler de post-islamisme ou de néo-communautarisme. 
D'après l'historique qu'en donnent Saint-Lary et Samson (2011) à partir d'une lecture principalement politique de la réislamisation dans les années 1990, liée surtout aux acteurs politiques islamistes et aux militants associatifs, les chercheurs se réorientent, à partir des années 2000, vers une lecture plus sociale et dépolitisée de la réislamisation, alors essentiellement perçue comme expérience individuelle et subjective. Des années 1990 aux années 2000, s'opère donc un basculement scientifique dans la manière d'appréhender la notion de réislamisation (Roy 2001). La dimension politique n'a pas nécessairement disparu du phénomène de réislamisation, tout comme l'expérience subjective de la réislamisation était déjà de mise chez les jeunes réislamisés des années 1990 (Khosrokhavar 1997). Tout semble dépendre du terrain de recherche et des acteurs religieux dont le chercheur rend compte ou du niveau d'analyse que ce dernier adopte pour aborder le phénomène. Dans le contexte français, certains, voire peut-être la plupart, des adeptes du renouveau islamique sont certes dépolitisés (Haenni 2006) ou tout du moins peu soucieux de politique, mais d'autres à l'heure actuelle demeurent au contraire dans le jeu politique (Fregosi 2008; Geisser 2012). De surcroît, si la réislamisation se vit bel et bien sur le mode de l'individualisme religieux, l'inscription individuelle dans le religieux passe par l'inscription dans une communauté croyante qui requiert une mise en conformité des discours, des pratiques et des normes.

D'après différents constats sociologiques (Roy 2004; Cesari 2004; Saint-Lary et Samson 2011), nous entendons donc par réislamisation, un mouvement islamique globalisé, porté notamment par la jeune génération de croyants, qui se diffuse par différents vecteurs ${ }^{2}$ et qui tend à moraliser le comportement des croyants, à apporter des solutions et des orientations pratiques dans leur vie quotidienne, et qui, en fonction des contextes étatiques et sociaux, tend à inscrire le religieux dans la sphère publique, voire politique. La réislamisation s'inscrit dans la sphère publique à travers, par exemple, les prêches religieux, l'organisation de meeting et de conférences religieuses, la construction d'édifices religieux ou, encore, la mise en place d'actions caritatives et éducatives. À ce titre, le renouveau islamique peut venir combler les défaillances des États, en matière sociale (Filakota 2009; Saint-Lary 2011); et en cela, s'il est globalisé, il n'en est, à notre sens, pas

2. Internet est à l'heure actuelle l'un des principaux moyens de diffusion du renouveau islamique (Bunt 2003; Roy 2004; Samson 2012). Toutefois, il se diffuse également par l'intermédiaire des flux migratoires et financiers, des médias télévisés et radiophoniques, des livres et cassettes audio, ainsi que des associations islamiques et des cours de religion. 
pour autant déterritorialisé (Sassen 2009): il continue de s'ancrer localement, en fonction des besoins des populations et des liens que les acteurs islamiques locaux nouent avec les pouvoirs publics.

Nous pouvons énumérer différents indicateurs qui, généralement, additionnés les uns aux autres, marquent l'inscription de l'individu croyant dans ce mouvement de réislamisation (Zhegal 2010; Saint-Lary et Samson 2011; Samson 2012). Celui-ci prône, d'une part, un retour à un islam "originel », par le biais de la lecture individuelle et directe des textes fondateurs ainsi que par l'exemple à suivre de la figure du prophète Mohammed. Suivre les traditions prophétiques et poursuivre une quête de savoir islamique est, en effet, l'incontournable devise des croyants réislamisés. D'autre part, ces derniers disent se conformer à un comportement «islamiquement correct ", en distinguant ce qui est licite de ce qui est illicite, en faisant référence à la vie dans l'au-delà et en luttant contre les innovations blâmables $($ bid'a). Ces réseaux dynamiques de croyance élaborent et affirment une identité islamique qui cherche constamment à distinguer culture et religion, à se détacher de certaines formes de l'islam dit "coutumier ", pour affirmer un islam universel et universalisable, balayant les éléments culturels qui viendraient l'entacher.

Ainsi, le mouvement général de réislamisation cherche à valoriser «l'unité de la foi de la communauté musulmane au-delà des différences sectaires [...] et des différences d'écoles juridiques» (Zhegal 2010, 1015). À ce titre, comme nous l'avons noté au cours de l'enquête, les croyants se refusent à revendiquer une appartenance islamique particulière. Si les femmes participent de la réislamisation contemporaine, elles ne se reconnaissent pas nécessairement dans un courant religieux spécifique. Quelques-unes se revendiquent d'un mouvement marocain proche des Frères Musulmans ${ }^{3}$, quelques autres du salafisme ${ }^{4}$, mais la plupart, à l'ère

3. L'égyptien Hassan al Banna (1906-1949) a fondé, en 1928, la confrérie des Frères Musulmans qui est venue poser les jalons de l'islamisme politique, en faisant du pouvoir politique l'un des piliers de la reconquête islamique (Carré et Michaud 1983). Le mouvement, qui a connu une large diffusion, est désormais implanté dans de nombreux pays. En France, né de l'exil de militants islamistes, ce mouvement s'incarne dans la structure associative de l'U.O.I.F. (Union des Organisations islamiques de France).

4. Puisant sa source dans l'interprétation doctrinale du théologien Ibn Taymiyya (12631328), le mouvement salafi se réclame des salaf, c'est-à-dire des compagnons du Prophète des premiers temps de l'islam. Les chercheurs (Adraoui 2013; Amghar 2011; Haenni 2006; Rougier 2008) distinguent deux types de salafisme: quiétiste (ou cheikhiste) et djihadiste. Les salafis djihadistes, minoritaires, sont idéologiquement 
du bricolage et de la diffusion virtuelle du religieux, puisent ici et là des références doctrinales qui leur conviennent (Jensen 2006). Toutefois, rappelons également que «cette volonté partagée de donner à voir un islam dégagé de toute division n'en cache pas moins une tendance généralisée à la réforme qui passe par la purification et la moralisation des mœurs » (Saint-Lary 2011, 231).

On pourrait aller plus loin et avancer, à l'instar de Saint-Lary (2012), qu'on assiste pour le dire vite à une "salafisation" des pratiques islamiques, en milieu réislamisé, ou en tout cas à un brouillage des frontières entre différents courants de la réislamisation plus ou moins radicaux. Ce que Saint-Lary démontre dans le contexte burkinabé semble, à notre sens, se vérifier dans des pratiques islamiques en France, bien que le contexte social soit différent et que l'islam dans ce cadre s'adresse surtout aux minorités. Il nous semble, sans que cela soit spécifiquement démontré par des recherches sociologiques qui viendraient conforter notre propos, qu'on assiste, dans les milieux de la réislamisation, à une diffusion-réappropriation de pratiques et conceptions religieuses, autrefois réservées aux adeptes du salafisme. Haenni (2006) parle de «banalisation du salafisme des autodidactes» (Haenni 2006, 131), à la suite de l'essoufflement des militants islamistes et du recul du mouvement Tabligh. Il note comment certains acteurs salafistes délaissent le parti-pris de condamner l'out-group pour, au contraire, s'ouvrir aux différents groupes islamiques qui œuvrent, à leur sens, pour le «Bien». Les frontières entre les différents mouvements de la réislamisation deviennent ainsi, d'après nous, plus poreuses; par conséquent, certaines pratiques ou certains marqueurs religieux, spécifiques à un islam salafi, s'installent progressivement dans le registre religieux ordinaire de pratiquants qui ne s'en revendiquent pourtant nullement.

\section{Le mariage halal ${ }^{5}$ en situation exogame: célébration de l'entre-deux et de l'entre-soi}

Le mariage, rite d'institution à la fois civil et religieux, va venir officialiser l'union sexuelle, généralement exogamique dans le cadre des conversions. Il procède à faire reconnaître aux yeux de l'État, des familles et de la communauté religieuse, l'alliance et la probabilité d'une descendance entre des

proches du mouvement Al-Qaïda, tandis que les salafis quiétistes défendent une vision apolitique de l'islam. De manière générale, les salafistes aspirent à rompre avec les normes dominantes de la société.

5. Cet intitulé fait écho au titre de l'article de Collet et Santelli (2012). 
individus issus de groupes socialement racisés et ethnicisés distincts. En France, légalement, le mariage civil l'emporte sur le mariage religieux et la loi prévoit même de sanctionner "tout ministre d'un culte qui procédera aux cérémonies religieuses de mariage sans que ne lui ait été justifié l'acte de mariage préalablement reçu par les officiers de l'état civil » (article 43321 du Code pénal). Ainsi, il arrive que les jeunes mariés aient fait le choix de célébrer leur mariage religieux avant le mariage civil, en l'absence d'un imam qui, en islam, n'est pas requis. Seulement, prenant modèle sur le déroulement du mariage chrétien, les jeunes pratiquants réinventent un rituel syncrétique, toutefois perçu comme spécifiquement islamique, où l'imam devient petit à petit, à l'image du prêtre, maître de cérémonie (Boubekeur 2003; Jouhanneau 2006). Lorsqu'ils le peuvent, les conjoints organisent, loin de leurs familles respectives, le mariage religieux dans l'idéal qui est le leur. Les jeunes mariés décident d'un mariage qui se veut simple, en présence de l'imam, de témoins et de quelques amis. Comme le suggèrent les propos de Mélanie (étudiante, 25 ans, deux enfants), rapidité, sobriété et intimité sont alors les maîtres-mots du déroulement des festivités:

E.: Tes parents étaient présents à ton mariage?

M.: Non, non. Le mariage civil, en mars de l'année d'après, oui. Je ne savais pas la date du mariage religieux, mon mari m'a dit qu'on allait probablement le faire la veille, mais je l'ai su le jour même. «Viens, il faut qu'on aille chercher des jus de fruit? - Pourquoi ? - Parce que l'imam arrive à $11 \mathrm{~h}$. — Quoi? » Il y en a qui ont des anniversaires surprises, moi c'était mariage surprise.

En petit comité, avec ou sans robe de mariée, le mariage a généralement lieu en semaine. «Je voulais pas un samedi, je voulais pas une robe blanche, donc on s'est mariés un mardi », nous explique Sabrina (mère au foyer, 26 ans, 2 enfants). Le choix de célébrer le mariage un autre jour que le samedi traditionnellement consacré au mariage dans la société française, et le refus de porter une robe blanche, marque ici une nette opposition aux normes majoritaires. Néanmoins, le port de la robe blanche et d'un voile blanc, ainsi que la séparation des sexes instituée lors de la célébration marque pour la jeune femme une renaissance symbolique dans la pureté qui accompagne sa conversion religieuse. Ce recours à la robe blanche, plutôt qu'à un autre vêtement traditionnel ou occidentalisé, montre également l'ajustement qui s'opère avec les normes majoritaires qui perdurent de manière euphémisée, en dépit de leur rejet. 
Par ailleurs, faisant fi des apparences et n'ayant que peu de ressources financières, les couples évitent des dépenses qu'ils estiment superflues. Le mariage coutumier, tel qu'il est pratiqué dans le pays d'origine ou par les familles immigrées, est critiqué pour être excessif et contraire aux volontés prophétiques. Streiff-Fenart notait déjà, en 1985, que les jeunes descendant de l'immigration maghrébine privilégiaient "l'utilité au détriment des dépenses de prestige " et préféraient "le mariage simple qui permet "d'acheter des meubles et la télé" au "grand mariage" ruineux " (StreiffFenart 1985, 130). Ces festivités frugales, un brin teintées d'austérité, traduisent l'inclusion dans un nouveau mode de vie prônant l'ascèse et une situation économique précaire, où les bas revenus des jeunes mariés ou des jeunes parents ne suffisent pas à organiser ces rituels dans le respect de la tradition coutumière qui requiert un budget économique relativement plus important.

Les jeunes pratiquants souhaitent se défaire de la matérialité qui entoure les rituels, tout en préservant une certaine convivialité, le plaisir de partager et de se retrouver entre amis. Les rituels sont plus ou moins dépossédés de leur caractère festif pour en faire un moment de partage non pas uniquement spirituel et extra-mondain, mais un moment de rencontre épuré de la dimension consumériste et traditionaliste faite de mises en scène orchestrées, de superstitions et de distinction sociale. La logique du don de soi à Dieu prime sur la logique classique de l'échange du don/ contre-don, où le rite a notamment pour fonction d'exposer le statut social des jeunes mariés et de sa famille aux yeux de l'entourage et du voisinage, en contrepartie de cadeaux et d'argent. Dans ces réseaux de jeunes croyants des Grands Ensembles, où le capital économique et la possession de biens matériels sont faibles, on préfère idéalement donner l'importance à l'hospitalité et à la spiritualité plutôt qu'aux cadeaux ou à l'argent distribué lors des rituels. On pourrait même avancer que la morale du renouveau islamique, dans une visée de dépassement des clivages sociaux et d'uniformisation des pratiques, tend idéalement à rompre avec la logique de don/ contre-don, qui sous-tend habituellement les pratiques rituelles et opère les distinctions sociales entre individus et groupes (Mauss 2012; Godbout 2000; Caillé 2007).

Toutefois, les femmes converties ne peuvent parfois se dispenser d'impliquer leurs belles-familles, lorsqu'elles résident en France, dans l'organisation du mariage. Elles tentent alors de freiner les dépenses, en limitant la durée du mariage à une seule journée et en évitant, par exemple, la location d'une salle ou l'intervention de musiciens, contraire selon elles à l'islam. 
Les femmes souhaitent également limiter le nombre d'invités, en fonction de la taille de l'appartement. La configuration des lieux nécessite parfois deux pièces distinctes, lorsque les conjoints procèdent à une séparation des sexes pour la réception des convives. Quant à la dot requise lors du mariage coutumier maghrébin, elle se résume pour ces femmes à un bijou ou à un euro symbolique. Voici comment s'est déroulé le mariage religieux de Samia, mariée à un immigré algérien dont les parents résident en France:

On a fait ça dans l'appartement de ma belle-famille. C'était pendant le repas du midi. Comme j'étais pas voilée, on voulait faire ça séparément. Par contre, comme l'approbation de la femme est obligatoire, mon tuteur me représentait auprès de l'imam dans la salle des hommes. Il y avait pas beaucoup de monde en fait. Au niveau des femmes, il y avait juste mes belles-sœurs, ma belle-mère, ma mère, ma sœur et deux, trois de mes amies. Par contre, mon mari avait invité plus de copains. Il y avait aussi mon beau-frère et mon papa. Alors, au début, c'est vrai que je voulais pas... Je voyais pas mes parents là-dedans, donc je ne les avais pas invités. Et, en fait, ma mère m'a fait comprendre qu'ils souhaiteraient bien être là, donc là, je me suis dit «comment ils vont réagir?» Finalement, ils sont venus. Je leur ai un peu dit comment ça allait se passer, mais pas assez... C'est vrai que ça leur a fait super bizarre d'être séparés. Du côté de mon père, c'était encore pire, parce que c'est chez eux que s'est déroulé le mariage religieux en fait, et je n'étais pas présente. C'est mon tuteur qui me représentait, c'était un ami converti, et mon papa comme il n'est pas musulman, ça ne peut pas être mon tuteur... On ne peut pas forcer une femme en islam, contrairement aux idées reçues, à se marier. Elle doit donner son consentement. Le tuteur, c'est vrai que ça fait un peu bizarre, mais il est là pour te représenter. Il y a la dot aussi. Pour la dot, j'ai demandé un euro symbolique. (Samia, sans emploi, 26 ans, un enfant)

Comme le démontre cet extrait d'entretien, les parents de femmes converties n'ont plus aucun rôle à jouer, si ce n'est celui de figurants, dans le mariage religieux, dont l'organisation est laissée aux mains des mariés et, plus rarement, de la famille musulmane. Ils sont généralement avertis, lorsqu'ils le sont, quelques jours avant ou la veille de la cérémonie religieuse, qui leur est présentée comme l'équivalent de fiançailles. Ecartés des préparatifs au mariage, leur présence, dans un environnement dont ils ne maîtrisent pas les codes, n'est pas toujours sollicitée. Ignorant le déroulement du mariage, c'est tout un monde, devenu celui de leur fille, que les parents découvrent, lorsqu'ils participent au mariage. Bien que certains parents, notamment les plus dotés en capitaux culturels, se soient toujours 
montrés ouverts aux pratiques culturelles maghrébines, comme ce fut le cas des parents de Samia, il s'agit, lors du mariage, d'accepter que sa propre fille prenne entièrement part à des rites culturels et cultuels qui leur sont étrangers. C'est principalement la séparation des sexes qui dérange profondément les familles. Le fait que la femme soit représentée par son tuteur ou walî, qui accepte le mariage en son nom (Rude-Antoine 1990), peut également déconcerter les pères qui se trouvent dans la salle des hommes. La langue, la gestuelle et les postures à tenir traduisent également le «décalage» ressenti par des parents qui s'interrogent sur le devenir de leur fille. Les parents ne sont pas toujours les seuls à ne pas maîtriser les codes du mariage maghrébo-musulman en contexte post-migratoire, les converti(e)s eux(elles)-mêmes peuvent se trouver déroutés par des pratiques inconnues. Lorsqu'elle se remémore son propre mariage, Sabine se souvient avoir assisté à un rituel dont elle ignorait le déroulement:

J'étais toute seule, il n'y avait personne de ma famille à ce moment-là. On était dans un appartement, chez son cousin, donc ils ont fait un grand repas. Ensuite, il y a l'imam qui est arrivé, on s'est mis dans une pièce et il a prononcé le mariage. Il a récité des sourates, donc là, par contre, j'étais complètement perdue. Je me disais: "Où je suis? Qu'est-ce que je fais là ? " J'étais complètement perdue ce jour-là. (Sabine, assistante maternelle, 34 ans, trois enfants)

Afin de compenser la mise à l'écart de la famille non musulmane, dans l'organisation et le déroulement du mariage religieux, les femmes souhaitent organiser, en contrepartie, un mariage civil, dans lequel leurs parents pourront s'impliquer davantage. Samira, par exemple, a tenu à ce que le mariage civil se déroule dans sa ville d'origine, alliant coutumes françaises et préceptes du renouveau islamique: robe blanche et port du voile, festivités sans alcool, mais avec musique d'ambiance.

Je disais à mon mari: "Je veux ma robe blanche, je veux ma robe blanche, je sais pas comment je vais faire pour le foulard... » Donc voilà, pareil, là ça s'est fait très vite. Je crois qu'on a décidé ça en mai et en septembre, on s'est mariés. On a fait ça dans le village de mes parents. On était une soixantaine de personnes, c'était pas séparé cette fois-ci. Il y avait de la musique pas trop, plutôt en musique de fond. On n'a pas trop dansé. Il y avait pas d'alcool. Ça c'était... on n'a même pas à réfléchir, c'est hors de question pour nous d'avoir de l'alcool à notre mariage. (Samira, assistante de direction, 30 ans, un enfant) 
Qu'il s'agisse de la célébration du mariage, de l'aqîqa ou de la circoncision, dans tous les cas, l'alcool est refusé et la viande halal instituée, ce qui ne va pas sans poser problème aux parents non musulmans. Les difficultés familiales proviennent généralement des choix alimentaires du couple respectueux de certains préceptes religieux et de leur refus de proposer des boissons alcoolisées durant les festivités. Choisir de ne pas proposer d'alcool lors d'une fête traditionnellement "arrosée ", signe véritablement l'inscription de la jeune mariée dans l'islam et dessine les limites de conciliation avec son héritage culturel.

\section{L'aqîqa : inscrire l'enfant dans la lignée croyante ou dans la lignée familiale?}

La cérémonie de l'aqîqa, considérée parfois comme une cérémonie de "baptême» ou plus justement comme une cérémonie d'entrée d'enfant dans la religion, a pour principale fonction d'inscrire l'enfant dans la lignée croyante. Comme le veut la tradition islamique, la cérémonie de l'aqîqa (en arabe classique) ou sboh (en arabe dialectal), tirée des enseignements du Prophète, est célébrée le septième, quatorzième ou vingt et unième jour après la naissance de l'enfant. Ce rituel varie d'un pays et d'une région à une autre, mais vise à protéger le nouveau-né et à le rapprocher de Dieu, dès sa venue au monde (Kadare 1997). À cette occasion, traditionnellement, une mèche de cheveux de l'enfant est coupée (son crâne peut être rasé) et un ou deux moutons sont sacrifiés. Dans les faits, par manque de moyens financiers, tous les convertis rencontrés ne font pas égorger de mouton(s) à la naissance de l'enfant. Celui-ci est souvent sacrifié «par procuration» (Brisebarre 2008). Autrement dit, l'argent est envoyé à la famille du conjoint étranger, restée au pays d'origine, qui se charge d'acheter et de faire égorger un ou deux moutons.

L'aqîqa est généralement présentée comme une occasion de se montrer généreux envers les pauvres. À l'image du mariage, la pratique de cette fête dans certains pays africains (notamment dans les pays d'Afrique subsaharienne), est critiquée pour son exubérance. Emblématique des aspirations du renouveau islamique, les convertis misent sur une fête simple où les convives sont reçus à leur domicile. Les grands-parents non musulmans ne sont en principe pas invités et les grands-parents musulmans, lorsqu'ils résident en France, peuvent être reçus à un autre moment que les amis. "Je mélange pas la famille de mon mari avec mes amies parce que, parmi mes amies, j'en ai qui ont porté le voile, la totale, et c'est vrai que j'ai une de 
mes belles-sœurs, ça passe pas du tout. Donc j'ai pas envie de les choquer ou de les embêter avec ça ", nous dit Pauline, assistante maternelle, 23 ans, deux enfants. Le parti pris de Pauline de ne pas mélanger son groupe d'amies à sa belle-famille manifeste concrètement la distinction qu'elle opère entre un islam d'élection, en l'occurrence ici un islam salafi, et un islam familial.

Un autre exemple de distinction opérée entre l'islam coutumier des grands-parents et l'islam d'élection des parents est la divergence de célébration de l'aqîqa entre les petits-fils de Geneviève. Geneviève, de milieu social modeste, a deux fils issus de deux précédents mariages avec des hommes non musulmans. Résidant en banlieue, son fils ainé s'est converti à l'islam à l'adolescence, à la suite de ses fréquentations de quartier et d'une socialisation à une culture de rue marquée par les référents islamiques. Il s'est assez rapidement rapproché du mouvement salafi, puis a épousé une jeune femme d'origine congolaise, également convertie à l'islam et en rupture avec son milieu familial d'origine. Plus tard, le fils cadet de Geneviève s'est converti à l'islam, à la suite, cette fois-ci, de son union avec une jeune fille d'origine maghrébine qu'il souhaitait épouser. Ce dernier, contrairement à son frère, ne pratique pas de manière intégrale sa religion. Durant cette même période de conversions de ses fils à l'islam, Geneviève vivait avec un homme musulman non pratiquant, de qui elle s'est séparée puisqu'il a été extradé vers la Tunisie. À la suite des difficultés conjugales qu'elle a connues et de cette séparation vécue comme brutale, Geneviève à son tour a fait le choix de l'islam.

La célébration de la naissance des petits-fils de Geneviève qui sont musulmans a donc varié en fonction de l'affiliation d'origine de leur mère. Pour l'enfant du fils aîné de Geneviève, «il y a pas eu de fêtes traditionnelles avec les tam-tams, tout le monde danse, non. Il y a eu Sunna, une fête obligée, un dû aux enfants ". En revanche, pour l'enfant de son fils cadet marié à une fille d'immigrés marocains, "c'était chez la maman de ma belle-fille qui a fait ça selon la tradition où on chante, où on danse » (Geneviève, assistante de vie scolaire, 50 ans). Dans le premier couple, composé de convertis, la volonté de "purifier» le rite, en le débarrassant du culturel pour ne plus en faire qu'un élément cultuel, est manifeste. Ni les chants ni les danses traditionnels ne sont cautionnés, seule importe la dimension sacrée de l'acte. Cette mise en pratique du rituel ne renvoie pas nécessairement à une inscription dans le mouvement salafi, on la retrouve également chez des pratiquants qui ne s'inscrivent explicitement dans aucun mouvement islamique dénommé, mais suivent un islam conservateur théologiquement bricolé. 
Néanmoins, leur islam se compose, entre autres, de recommandations et de pratiques habituellement entretenues par le mouvement salafi.

Certaines femmes, comme Sabine (qui nous a précédemment dit avoir célébré son mariage sans en maîtriser le déroulement), refusent de composer avec les attentes familiales d'ordre coutumier. Traditionnellement, au Maghreb, l'aqîqa peut s'accompagner de rites de protection de l'enfant, c'est-à-dire de pratiques pour le protéger du monde invisible (Touhami 2010), qui varient selon les pays et les régions. Le refus des femmes converties de suivre ces coutumes manifeste le contrôle qu'elles exercent à leur tour sur la descendance et questionne des traditions qui relèvent, pour elles, plus de la superstition que de la religion. Les femmes converties repoussent les pratiques coutumières qu'elles estiment plus culturelles que cultuelles pour seulement suivre les règles édictées par le Coran et la Sunna.

Sur un plan plus culturel, dans le village tunisien de mon mari, ils enroulent l'enfant dans une espèce de linge avec des herbes, ils mettent des pétales de fleur sur les joues des petites filles et ça, j'ai toujours refusé de le faire. Je trouve ça immonde de mettre plein d'herbes sur un petit bébé. Après, quand ils l'enlèvent, ils disent que s'il a fait sur lui, il va être toujours sale. Pour moi, c'est complètement débile. Pour la grande, comme ma belle-mère était pas là, j’ai acheté une vareuse, je lui ai mis deux pétales sur la joue pour faire semblant. J'ai fait une photo et comme ça, elle était contente. Pour les deux autres, je l'ai pas fait. (Sabine)

Par ailleurs, à propos de la cérémonie de l'aqîqa, beaucoup admettent qu'il est religieusement requis d'égorger un mouton pour une fille et deux pour un garçon. Les écoles juridiques ${ }^{6}$ sont divisées à ce sujet: pour les écoles hanafite et malékite, quel que soit le sexe de l'enfant, un seul mouton suffit, tandis que pour les écoles hanbalite ${ }^{7}$ et chaféite, il est nécessaire d'égorger deux moutons à la venue d'un garçon. Quoi qu'il en soit, lorsque j'interroge les points de vue des femmes converties sur cette pratique, à savoir si elles ne la perçoivent pas comme discriminatoire vis-à-vis du sexe

6. Dans l'islam sunnite, il existe quatre écoles de jurisprudence islamique ( $f i q h)$, dans l'ordre de leur apparition: le Hanafisme (de Abû Hanifâ, 700-767); le Malékisme (de Malîk Ibn Anas, 712 et 796); le Shafi'isme (de Al-Shafi'i, 768-820); le Hanbalisme (de Ibn Hanbal, 781-856). «Le Hanafisme s'est massivement implanté en Turquie et en Asie centrale, le Mâlikisme au Maghreb, le Shafi'isme au MoyenOrient et en Indonésie, alors que le Hanbalisme a toujours été très minoritaire, sinon, à certaines époques, en Syrie» (Chaumont 2010, 293).

7. L'école hanbalite est généralement considérée comme la plus moralisante de toutes (Chaumont 2010). 
féminin, elles m'expliquent qu'il ne s'agit pas de la manifestation d'une inégalité entre fille et garçon, mais d'un rituel venant pointer la future responsabilité de l'homme. En arguant de la complémentarité des rôles entre les sexes, Sandrine (sans emploi, 28 ans, 3 enfants) vient restituer l'«égalité » non perceptible de cette pratique:

C'est pour montrer que le garçon aura une lourde charge sur ses épaules! En islam, l'homme doit tout à sa femme, sa famille. Il doit la nourrir, la loger, l'habiller. La femme ne lui doit rien, lui en plus paye la dot. C'est là que réside la différence.

L'aqîqa, tout comme la circoncision, participe ainsi à inscrire l'enfant dans la sphère masculine ou féminine.

\section{La circoncision : faire reconnaître et se reconnaître dans la descendance}

Dans certains pays musulmans, la circoncision est désignée par le terme tahara qui évoque, en arabe, la pureté ou l'hygiène (Chebel 2000). À propos de la circoncision, dans la tradition juive, Fellous (2001) écrit qu' «elle opère le passage de la nature à la culture. Le rite transforme le corps matière en corps humanisé, socialisé " (Fellous 2001, 26). La circoncision est ici une question relativement peu discutée au sein des couples. Signe d'appartenance à la communauté de croyants, elle va de soi pour une majorité d'entre eux. Seules Alice et Julie, attachées à une pratique plus textuelle que culturelle, questionnent sa validité religieuse, puisqu'elle n'est pas directement recommandée par le Coran, mais se réclame de la Sunna ${ }^{8}$. Elles entendent étudier le sens de la circoncision, à partir d'avis juridiques qui débattent également du caractère obligatoire ou simplement recommandé de la circoncision (Kamkar-Rad 2004). Voici ce qu'en dit Julie:

C'est une pratique que font les musulmans, mais c'est pas une pratique qu'est... C'est pas dans le Coran. C'est pareil, il faut savoir pourquoi on le fait: si c'est une tradition ou est-ce que ça a vraiment un sens. Maintenant le Coran, il parle pas du tout de la circoncision, mais vu que tout est une continuité: est-ce qu'il faudrait continuer à faire ça ou pas? Je me suis déjà posé la question, parce que j'ai une amie qui a des fils qui sont pas circoncis. On s'est renseigné à savoir si c'était une obligation ou pas. Les mamas traditionnelles, elles vont dire: "Oui, les enfants sont pas circoncis mais c'est pas des musulmans!» alors qu'il y a pas... Dans le Coran, quand il parle du

8. La Sunna renvoie à la tradition prophétique relatée dans les recueils de hadiths. 
baptême, il parle pas de la circoncision. Il parle de faire un sacrifice d'un mouton, mais pas de circoncision. Pour moi, c'est une pratique traditionnelle. (Julie, auxiliaire de vie, 25 ans, sans enfant)

À travers le discours que tient Julie, on voit bien comment les femmes converties sont prises entre le refus d'une tradition culturelle et la continuité religieuse, entre le refus d'un acte douloureux et le désir de ne pas priver leur fils de l'histoire de leur père. «Mais, ce serait difficile pour mon mari d'imaginer que ses fils ne soient pas circoncis " (Alice, éducatrice spécialisée, 25 ans, sans enfant). En effet, les hommes semblent tous en manifester le souhait, y compris les moins pratiquants. Plus que la religion, la ressemblance au père et la perpétuation de la filiation patrilinéaire semblent être les motivations premières de la circoncision. Mathieu (2009) et Tank-Storper (2007) perçoivent la pratique de la circoncision, au sein de couples mixtes juifs/non-juifs, comme un processus assurant non seulement les continuités masculines, mais opérant le passage de l'ordre du féminin à l'ordre du masculin. Il s'agit d'un dispositif symbolique qui participe à l'intégration de «l'enfant dans le monde masculin de la culture en l'arrachant à l'ordre féminin de la nature» (Tank-Storper 2007, 117). À propos du rite de circoncision, Bourdieu (1982) écrit qu'il permet de tracer une démarcation symbolique entre un avant et un après, entre les hommes qui l'ont subi et ceux qui ne l'ont pas subi, et enfin et principalement entre les hommes et les femmes.

L'effet majeur du rite est celui qui passe le plus complètement inaperçu: en traitant différemment les hommes et les femmes, le rite consacre la différence, il l'institue, instituant du même coup l'homme en tant qu'homme, c'est-à-dire circoncis, et la femme en tant que femme, c'est-à-dire non justiciable de cette opération rituelle. (Bourdieu 1982, 58)

En cela, Bourdieu préfère parler de rite d'institution, au sens où le rite de passage institue et fait reconnaître comme légitime et naturelle une différence socialement construite, ici la différence sexuée. La turcologue Sauner-Leroy (2008) démontre également que, dans la société turque, une des principales fonctions de la circoncision, puis du mariage, est d'instituer une différence sociale entre hommes et femmes, et de définir la place des sexes dans la reproduction. Ainsi, l'identité sexuelle est pensée en lien avec la procréation et se construit socialement à partir de sa ritualisation.

La circoncision permet ainsi au jeune garçon de quitter la sphère féminine tout en l'instituant comme homme et musulman. Dans le cas du mariage, il s'agira de même d'instaurer un homme et une femme en tant que couple 
marié, le groupe social les autorisant publiquement à avoir accès à la sexualité et à la reproduction. (Sauner-Leroy 2008)

De ce que nous avons pu constater, les pères musulmans, pratiquants ou non, souhaitent profondément transmettre à travers cet acte une part d'eux-mêmes. La circoncision contribue ainsi à inscrire l'enfant dans la sphère du masculin et à renforcer le lien entre le père et sa descendance, d'une part, et entre les hommes du groupe, d'autre part. Mais c'est toujours au nom de l'hygiène qu'est légitimée la pratique de la circoncision:

Alors maintenant, il y a le côté hygiénique. On pense que c'est plus hygiénique aussi de circoncire les enfants. C'est plus propre. Je pense que si on fait ça, il faut le faire à un enfant qui est assez jeune parce qu'après, il peut y avoir des traumatismes. Je pense que ça doit être une opération douloureuse donc ça peut traumatiser un enfant. Moi, ça sera vraiment en fonction du mari. Ça, j'en ai pas discuté encore avec l'homme que je fréquente, mais ça sera en fonction de la tradition ou de... Après on se met d'accord. (Julie, auxiliaire de vie, 25 ans, sans enfant)

Les justifications hygiénistes de la pratique de la circoncision sont incontournables chez les couples. Mathieu (2009) avance à ce propos que légitimer la circoncision par l'hygiène permet de dépasser la mutilation du corps par la rationalisation du geste, le dépossédant ainsi de son «archaïsme». En ce qui concerne l'âge à la circoncision", les femmes préfèrent qu'elle ait lieu avant l'âge de deux ans pour, disent-elles, ne pas blesser ni traumatiser l'enfant. Le registre lexical de la souffrance et de la peur est récurrent: "J'ai tellement peur avec le fait de penser qu'il va pouvoir souffrir. Je pense que je vais le faire, mais c'est dur de se dire que son fils va souffrir» (Marlène, 26 ans, un enfant). En revanche, les maris préfèrent attendre l'âge de quatre ou cinq ans, âge auquel ils ont eux-mêmes reçu la circoncision. Ils souhaitent faire de ce rituel un véritable rituel initiatique qui s'inscrit dans le corps et la mémoire du jeune garçon et perpétue par là même la mémoire du groupe. En signe de compromis, et à l'exception de quelques couples salafi qui pratiquent la circoncision le jour de l'aqîqa, c'est souvent entre l'âge d'un et deux ans que les garçons sont

9. En islam, l'âge de la circoncision ne fait pas l'unanimité entre les juristes: certains recommandent de la faire à sept jours ou au contraire, de ne surtout pas la faire à sept jours pour se différencier de la coutume juive. Pour d'autres, elle peut avoir lieu à l'âge d'un mois ou d'un an; d'autres encore préconisent d'attendre l'âge de cinq, sept ou dix ans (Kamkar-Rad 2004). 
opérés ${ }^{10}$. L'intervention a souvent lieu, pour les couples franco-maghrébins, dans le pays d'origine du conjoint et est célébrée en famille. Lorsqu'elle a lieu en France, elle se déroule à l'hôpital ${ }^{11}$ ou au sein de cabinets médicaux spécialisés dans ce type d'opération.

\section{Conclusion : ritualités entre continuité et discontinuité}

L'étude de la conversion islamique, dans le cadre des productions rituelles, et vice versa, nous a permis de détailler les productions et reproductions religieuses et identitaires qui ont cours. Se faisant des passeuses de frontières (Mossière 2010), des garantes de la Tradition, les femmes converties transmettent à leur façon des coutumes ancestrales aux futures générations. Témoignant d'un nouveau rapport symbolique et pratique au monde, elles réinventent les rites de passage entre pratiques coutumières, religiosités éprouvées et collectivement partagées.

C'est en bricolant, à partir de traditions culturelles et familiales diverses, voire contradictoires, mais aussi et surtout en donnant part à la nouvelle culture morale religieuse, que les rituels sont agencés et les figures d'autorité inversées. Les rites présentés sont célébrés dans un entre-soi qui exclut la famille non musulmane et tolère avec compromis l'implication de la famille musulmane. Si, de manière inhérente, ces rites demeurent collectifs, ils ne sont plus nécessairement familiaux. Ils fonctionnent en «petits » rassemblements communautaires, tenant à distance, voire à l'écart, la famille, sans pour autant s'autonomiser totalement. Plus que d'une intégration familiale, ils sont vecteurs d'une intégration au groupe de pairs, c'est-à-dire à la communauté de jeunes musulmans pratiquants qui participe à faire évoluer les modalités du rituel.

\section{Références}

Adraoui, M.-A. (2013), Le salafisme, du Golfe aux banlieues, Paris, PUF. Amghar, S. (2011), Le salafisme d'aujourd'hui. Mouvements sectaires en Occident, Paris, Michalon.

Arslan, L. (2010), Enfants d'Islam et de Marianne, Paris, PUF.

10. La pratique chirurgicale de la circoncision au laser, sous anesthésie locale, se développe au sein des cabinets médicaux.

11. En France, la circoncision rituelle médicalisée est tolérée au sein des hôpitaux publics, mais non légiférée (Meddour 2005). 
Boubekeur, A. (2003), «Le jeune marché matrimonial musulman en France, un espace entre spontanéité et institutionnalisation", Confluences Méditerranée, 46, p. 157-167.

Bourdieu, P. (1982), "Les rites comme actes d'institution", Actes de la recherche en sciences sociales, 43, p. 60-63.

Brisebarre, A.-M. (2008), "Conflits autour de l'Aïd el Kebir en France», dans A.-S. Lamine, F. Lautman et S. Mathieu, dir., La religion de l'autre: la pluralité religieuse entre concurrence et reconnaissance, Paris, L'Harmattan, p. 199-209.

Bunt, G. (2003), Islam in the Digital Age. E-Jihad, Online Fatwas and Cyber Islamic Environments, London, Pluto Press.

Carre, O. et Michaud, G. (1983), Les frères musulmans: 1928-1982, Paris, Gallimard.

Caille, A. (2007) [20001], Anthropologie du don: le tiers paradigme, Paris, La Découverte.

Cesari, J. (2004), L'islam à l'épreuve de l'Occident, Paris, La Découverte.

Chaumont, E. (2010), «Écoles juridiques en Islam», dans D. HervieuLÉger et R. Azria, dir., Dictionnaire des faits religieux, Paris, PUF, p. 291-294.

Chebel, M. (2000), Dictionnaire des symboles musulmans: rites, mystique et civilisation, Paris, Albin Michel.

Collet, B. et Santelli, E. (2012), "Le mariage "halal”. Réinterprétation des rites du mariage arabo-musulman dans le contexte post-migratoire français ", Recherches familiales, 9, p. 83-92.

Fellous, M. (2001), À la recherche de nouveaux rites. Rites de passage et modernité avancée, Paris, L'Harmattan.

Filakota, R. (2009), Le renouveau islamique en Afrique noire. L'exemple de la Centrafrique, Paris, L'Harmattan.

Fregosi, F. (2008), «Les nouveaux contours du champ intellectuel musulman en France", Revue des mondes musulmans et de la Méditerranée, 123, p. 93-115.

Geisser, V. (2012), «La "question musulmane" en France au prisme des sciences sociales. Le savant, l'expert et le politique ", Cabiers d'études africaines, 206-207, p. 351-366.

Godbout, J. (2000), Le don, la dette et l'identité. Homo donator versus homo œeconomicus, Montréal, La Découverte/Boréal. 
Gooren, H. (2007), « Reassessing Conventional Approaches to Conversion. Toward a New Synthesis ", Journal For The Scientific Study of Religion, 46/3, p. 337-353.

Haenni, P. (2006), «La France face à ses musulmans. Émeutes, jihadisme et dépolitisation ", Esprit, Octobre, p. 112-145.

Jensen, T. (2006), "Religious Authority and Autonomy Interwined. The Case of Converts to Islam in Denmark ", The Muslim World, 96, p. 643-660.

JouAnneau, S. (2006), «L'imam et les jeunes mariés. Élaborer en France de nouvelles normes islamiques en matière de gestion du marché matrimonial ", Communication à l'ENS, Paris; disponible sur: <http://barthes. enssib.fr/clio/seminaires/himmig/jouanneau.pdf> (consulté le 30 mai 2010).

Kadare, I. (1997), dir., Dictionnaire de l'islam. Religion et civilisation, Paris, Encyclopédie Universalis/Albin Michel.

KAMKAR-RAD, Q. (2004), "La circoncision, rite chirurgical, chirurgie rituelle», Droit, déontologie et soin, 4/2, p. 209-225.

Khosrokhavar, F. (1997), L'islam des jeunes, Paris, Flammarion.

Mauss, M. (2012) [1902], Essai sur le don. Forme et raison de l'échange dans les sociétés archaïques, Paris, PUF.

Mathieu, S. (2009), La transmission du judaïsme dans les couples mixtes, Paris, Éditions de l'Atelier.

Meddour, S. (2005), "Soin et respect des croyances ", Droit, déontologie et soin, 5/2, p. 148-182.

Mossiere, G. (2010), «Passer et retravailler la frontière... Des converties à l'islam en France et au Québec: jeux et enjeux de médiation et de différenciation ", Sociologie et sociétés, 42/1, p. 245-270.

Piette, A. (2002), Le fait religieux. Une théorie de la religion ordinaire, Paris, Economica.

Rougier, B. (2008), Qu'est-ce que le salafisme?, Paris, PUF.

Roy, O. (2001), «Les islamologues ont-ils inventé l'islamisme?», Esprit, 277, p. 116-138.

(2004), L'Islam mondialisé, Paris, Seuil.

Paris, Seuil.

(2008), La sainte ignorance. Le temps de la religion sans culture,

Rude-Antoine, E. (1990), Le mariage maghrébin en France, Paris, Karthala. 
Saint-Lary, M. (2011), "J'épargne pour l'Au-delà ". Le salut comme rhétorique de la réislamisation au Burkina Faso ", L’Homme, 198-199, p. 227-246.

(2012), «Du Wahhabisme aux réformismes génériques. Renouveau islamique et brouillage des identités musulmanes à Ouagadougou ", Cahiers d'études africaines, 206-207, p. 449-470.

Saint-Lary, M. et Samson, F. (2011), «Pour une anthropologie des modes de réislamisation. Supports et pratiques de diffusion de l'islam en Afrique subsaharienne ", Ethnographiques, 22; disponible sur <http://www.ethnographiques.org/2011/Saint-Lary,Samson> (consulté le 21/01/2014).

Samson, F. (2012), "Les classifications en islam ", Cahiers d'études africaines, 206-207, p. 329-349.

Sassen, S. (2009), La globalisation. Une sociologie, Paris, Gallimard.

Sauner-Leroy, M.-H. (2008), "La circoncision, le mariage et le corps. Une blessure en partage ", Journal des anthropologues, 112-113, p. 47-74; disponible sur <http://jda.revues.org/687> (consulté le 21/01/14).

Seniguer, H. (2012), "Les catégories dénominatives de l'islam à l'épreuve d'un objet "mutant". Le cas du Parti de la justice et du développement marocain ", Cahiers d'études africaines, 206-207, p. 603-633.

Simon, P. et TiberJ, V. (2013), "Sécularisation ou regain religieux. La religiosité des immigrés et de leurs descendants ", Documents de travail, INED, 196.

Streiff-Fenart, J. (1985), "Le mariage. Un moment de vérité de l'immigration familiale maghrébine ", Revue européenne des migrations internationales, 1, p. 129-141.

Tank-Storper, S. (2007), Juifs d'élection. Se convertir au judaïsme, Paris, Éditions du CNRS.

Tounami, S. (2010), La part de l'œil. Une ethnologie du Maghreb en France, Éd. CTHS.

Zhegal, M. (2010), "Réformismes et réveils en islam ", dans D. HervieuLÉger et R. Azria, dir., Dictionnaire des faits religieux, Paris, PUF, p. 1012-1017. 


\section{Résumé}

La réappropriation de rites de passage islamiques que sont le mariage, l'aqîqa et la circoncision, par des femmes converties à l'islam, marque leur inscription dans un nouveau groupe d'appartenance. La mise en conformité de rituels traditionnels avec les exigences du renouveau islamique contemporain, tel qu'il prend forme en France, en sus de la négociation avec une culture héritée non islamique qu'elles mettent en œuvre, s'apparente à un agencement de l'entre-deux qui participe, d'une part, à leur inclusion sociale au sein de la communauté de croyants et, d'autre part, à produire une nouvelle éthique religieuse. Toutefois, à la suite d'une volonté marquée de déculturer le religieux, de se distancier de rites perçus comme coutumiers et non valides sur le plan religieux, la fonction de rassemblement des ritualités se limite à un entre-soi religieux, car une mise à distance, voire une mise à l'écart des familles s'opère.

\section{Summary}

The re-appropriation of Islamic rites of passage such as marriage, aqiqa and circumcision by women having converted to Islam denotes their belonging to a new social group. Making traditional rituals comply with the requirements of French-style contemporary Islamic revival, in addition to the necessary negotiations with inherited non-Islamic culture, is a sort of "inbetween" arrangement. On the one hand, it contributes to their inclusion in the community of believers, and on the other hand, it forges a new religious ethic. However, given the determination to «deculturize» religion, to create a distance from rituals perceived as traditional, superstitious and not valid from a religious point of view, the function of these rituals that gather people together is simply that of a religious group identity, for families are kept at a distance, if not completely excluded. 